\title{
Comparative Effects of Toll-Like Receptor Agonists on a Low Dose PspA Intranasal Vaccine against Fatal Pneumococcal Pneumonia in Mice
}

\author{
Zhenyu Piao ${ }^{1,2}$, Keita Oma ${ }^{1,3}$, Hirokazu Ezoe ${ }^{1}$, Yukihiro Akeda ${ }^{1}$, Kazunori Tomono ${ }^{2}$ and Kazunori Oishi ${ }^{1 *}$ \\ ${ }^{1}$ Laboratory for Clinical Research on Infectious Diseases, International Research Center for Infectious Diseases, Research Institute for Microbial Diseases, 3-1 \\ Yamadaoka \\ ${ }^{2}$ Division of Infection Control and Prevention, Osaka University Graduate School of Medicine, 2-15 Yamadaoka, Suita, Osaka 565-0871 \\ ${ }^{3}$ OK Pharmacy, Nagasaki, Nagasaki 850-0841, Japan
}

\begin{abstract}
To develop a cost-effective pneumococcal vaccine, we compared the effects of a panel of Toll-like receptor (TLR) agonists on a low dose pneumococcal surface protein $A(P s p A)$ nasal vaccine in a fatal pneumococcal pneumonia model using a serotype 3 strain. The mice were nasally immunized with $10 \mu \mathrm{g}$ of the TLR agonist (TLR 2, 3, 4 and 9) and $0.1 \mu \mathrm{g}$ of PspA once per week for three weeks. A high level of PspA-specific immunoglobulin G (IgG) was detected in sera of mice that were nasally administered a low dose of PspA plus each TLR agonist, while no PspAspecific IgG were detected in sera of mice that had been nasally administered a low dose of PspA alone. A relatively low level of PspA-specific lgG was also detected in the airway of mice that had been nasally administered a low dose of PspA plus each TLR agonist. The binding of PspA-specific IgG increased the deposition of C3 on the bacterial surface. Bacterial density in the lung and blood was significantly decreased in mice that had been administered a low dose of PspA plus each TLR agonist, compared with mice that received a low dose of PspA alone $24 \mathrm{~h}$ after a bacterial challenge. Furthermore, significant increases in survival rate were found in a murine model of fatal pneumonia that had been nasally administered a low dose of PspA plus each TLR agonist, compared with mice that received a low dose of PspA alone. The rank order of TLR agonists on the effect of increasing survival rate was LPS > Pam3CSK4 > Poly(I:C) and CpG 1826. These data suggest a potentially new strategy for the development of a cost-effective intranasal vaccine with a low dose PspA plus TLR agonist that would be effective against lifethreatening bacteremic pneumococcal pneumonia.
\end{abstract}

Keywords: PspA; TLR agonist; Intranasal vaccine; Streptococcus pneumoniae; Pneumonia

Abbreviations: PspA: Pneumococcal surface protein A; TLR: Toll-Like Receptor; LPS: Lipopolysaccharide; Poly(I:C): Polyinosine-polycytidylic acid; CpG ODN 1826: CpG-Containing Oligodeoxynucleotide 1826; BALF: Bronchoalveolar lavage fluid; NW: Nasal wash

\section{Introduction}

S. pneumoniae is a leading human pathogen that causes a wide variety of diseases, ranging from otitis media to pneumonia, bacteremia, and meningitis in both children and adults. Pneumococcal infections can occur at any age but are more frequent in infants, the elderly and immunocompromised patients. Despite the development of effective treatments, the pneumococcus has remained a significant cause of morbidity and mortality worldwide [1,2]. Because of this, a clear need for an effective vaccine for the prevention of disease exists. Currently licensed polysaccharide-based pneumococcal vaccines only elicit protective antibodies against the infection of serotypes that are included in the vaccine. In addition, invasive diseases attributable to non-vaccine serotypes of $S$. pneumoniae have increased greatly $[3,4]$. Therefore, the search for new vaccine candidates that elicit protection against a broader range of pneumococcal strains is an important goal. To broaden the protection, the use of pneumococcal proteins represents a feasible and preferable alternative. Several pneumococcal proteins are currently under investigation as potential candidates for such a vaccine $[5,6]$. One of these proteins, PspA has recently undergone phase one clinical trials in humans and has been found to be safe and highly immunogenic $[7,8]$. PspA is a surface protein of $S$. pneumoniae that is found on all pneumococci and is broadly expressed among different serotypes of pneumococci [8]. Antibodies to PspA generated in mice $[9,10]$ or humans $[7,8]$ are capable of passively protecting mice against infections with different serotypes. PspA is, therefore, an attractive candidate for use as future protein-based pneumococcal vaccines.

Since S. pneumoniae enters the host primarily through the respiratory mucosa, vaccination strategies designed to target the airways are of great interest. An appropriate mucosal adjuvant is required to elicit an antigen-specific immune response in both the mucosal and systemic compartments [11]. We previously reported that each of the TLR agonists used in this study is an effective nasal adjuvant for the PspA antigen at a high dose ( $2.5 \mu \mathrm{g}$ per mouse), and that it elicited the production of PspA-specific IgA in the airways and PspA-specific IgG in plasma. Because of this, it was capable of reducing the bacterial load in both the nasopharynx and lungs after a challenge with pneumococci with different serotypes [12]. Nasal immunization with a high dose of PspA alone could induce a certain level of PspA-specific IgG in the plasma and increased mouse survival, compared with mice that were nasally immunized with PBS alone, in a fatal pneumonia model in this study. These data suggest that nasal immunization with the reduced

*Corresponding author: Kazunori Oishi, Laboratory for Clinical Research on Infectious Diseases, International Research Center for Infectious Diseases, Research Institute for Microbial Diseases, Osaka University, 3-1 Yamadaoka, Suita, Osaka 565-0871, Japan, Tel: 81-6-6879-4253; Fax: 81-6-6879-4255; E-mail: oishik@biken.osaka-u.ac.jp

Received January 28, 2011; Accepted March 14, 2011; Published March 19 2011

Citation: Piao Z, Oma K, Ezoe H, Akeda Y, Tomono K, et al. (2011) Comparative Effects of Toll-Like Receptor Agonists on a Low Dose PspA Intranasal Vaccine against Fatal Pneumococcal Pneumonia in Mice. J Vaccines Vaccin 2:113. doi:10.4172/2157-7560.1000113

Copyright: (c) 2011 Piao Z, et al. This is an open-access article distributed under the terms of the Creative Commons Attribution License, which permits unrestricted use, distribution, and reproduction in any medium, provided the original author and source are credited. 
dose of PspA in combination with a TLR agonist was able to prevent the development of fatal pneumonia in this model. In the present study, we therefore examined the issue of whether nasal immunization using different TLR agonists in conjunction with a low dose $(0.1 \mu \mathrm{g}$ per mouse) of PspA could confer protection against fatal pneumococcal pneumonia in healthy mice.

\section{Materials and Methods}

\section{Mice and bacterial strains}

Female C57BL/6 mice (6- to 8-wk-old) were purchased from Charles River Japan, Kanagawa, Japan. Mice were transferred to microisolators and maintained in horizontal laminar flow cabinets. They were provided sterile food and water in a specific pathogenfree facility. All mice used in these experiments were free of bacterial and viral pathogens. All animal experiments described in this study (protocol number; 08008) were performed in accordance with institutional guidelines for the Osaka University animal facility. $S$. pneumoniae WU2 strain with serotype 3, expressing PspA belonging to family 1, clade 2, was grown in Todd-Hewitt Broth (BD, Franklin Lakes, $\mathrm{NJ}$ ) supplemented with $0.1 \%$ yeast extract (THY) to the mid-log phase and washed twice with phosphate-buffered saline (PBS) without $\mathrm{CaCl}_{2}$ and $\mathrm{MgCl}_{2}$. Bacteria were suspended in THY, and aliquots were snap frozen in liquid nitrogen and stored at $-80^{\circ} \mathrm{C}$ until used.

\section{Recombinant PspA and adjuvants}

Recombinant PspA/Rx1 (amino acids 1 to 302) was prepared as previously described [8]. To extensively remove LPS from the PspA preparations, we used an LPS removal column, ProteoSpin ${ }^{\mathrm{R}}$, (Norgen, Thorold, Canada). Four TLR agonists, including Escherichia coli K12 LPS (TLR4 agonist), Pam3CSK4 (TLR1/2 agonist), Poly(I:C) (TLR3 agonist) or CpG ODN 1826 (TLR9 agonist) were selected to use as adjuvants. The LPS, Pam3CSK4 and Poly(I:C) were purchased from InvivoGen (san Diego, CA). CpG ODN 1826 was purchased from Hokkaido System Science (Sapporo, Japan). Each of these adjuvants was used in a dose of $10 \mu \mathrm{g}$ for nasal immunization.

\section{Nasal immunization}

Mice were immunized intranasally three times at weekly intervals with $12 \mu \mathrm{l}$ of PBS containing $10 \mu \mathrm{g}$ of each TLR agonist and $0.1 \mu \mathrm{g}$ of PspA, $0.1 \mu \mathrm{g}$ of PspA alone or $12 \mu \mathrm{l}$ of PBS on days 0 , days 7 and days 14 . The mice were euthanized on day 21 to obtain serum, bronchoalveolar lavage fluid (BALF) and a nasal wash (NW). The time points of nasal immunization and sampling for the determination of antibody levels were determined according to our previous study [13]. After removing the mandible, the nasal cavity was gently flushed with $1 \mathrm{ml}$ of PBS from the posterior opening of the nose. The NW obtained from the anterior openings of the nose was collected. BALF was obtained by irrigation with $1 \mathrm{ml}$ of PBS using a blunted needle inserted into the trachea after a tracheotomy [13]

\section{PspA-specific antibody assays}

PspA-specific antibody titers of IgG or IgA in Serum, BALF and NW were determined by ELISA as previously described [12]. PspA was used as the coating antigen $(1 \mu \mathrm{g} / \mathrm{ml})$. A $100 \mu \mathrm{l}$ sample was added to each well, followed by incubation at $37^{\circ} \mathrm{C}$ for $30 \mathrm{~min}$. The plate was washed, and then reacted with $100 \mu$ l of alkaline phosphatase-conjugated goat anti-mouse IgA, IgG, IgG1 or IgG2a (Zymed, San Francisco, CA) and the $\mathrm{OD}$ at $405 \mathrm{~nm}$ was then measured. End-point titers were expressed as the reciprocal $\log _{2}$ of the last dilution giving an $\mathrm{OD}_{405}$ of $0.1 \mathrm{OD}$ unit above the $\mathrm{OD}_{405}$ of negative control samples obtained from nonimmunized mice.

\section{IgG binding and C3 deposition assays}

Antibody binding was analyzed by whole cell ELISA. Frozen stock of S. pneumoniae WU2 (family 1 and clade 2) were plated onto blood agar, incubated overnight and then grown in THY to an $\mathrm{OD}_{600}$ of $0.4 \sim 0.5$ and harvested by centrifugation. The bacterial cells were washed, resuspended in PBS, and fixed with $80 \%$ ethanol at room temperature for $1 \mathrm{~h}$. The ethanol-killed bacteria were washed twice with PBS, and the pellet resuspended in PBS to an $\mathrm{OD}_{600}$ of about 0.2. $50 \mu \mathrm{l}$ of the ethanol-killed bacteria were coated in ELISA overnight at $4^{\circ} \mathrm{C}$. The following day, the wells were washed 3 times with $0.05 \%$ of Tween-20 in PBS (PBST). The plates were then blocked with $3 \%$ of skim milk in PBST at room temperature for $1 \mathrm{~h}$. After 3 washes with PBST, $50 \mu$ of diluted serum in $1 \%$ of skim milk in PBST were added to the plates, which were then incubated at $37^{\circ} \mathrm{C}$ for $2 \mathrm{~h}$. The plates were washed 3 times with PBST, and then reacted with $100 \mu$ of alkaline phosphatase-conjugated goat anti-mouse IgG (Zymed, San Francisco, $\mathrm{CA})$. The OD at $405 \mathrm{~nm}$ was then measured. The end-point titers were expressed as the reciprocal $\log _{2}$ of the last dilution giving an $\mathrm{OD}_{405}$ of $0.1 \mathrm{OD}$ unit above the $\mathrm{OD}_{405}$ of negative control samples obtained from non-immunized mice.

C3 deposition was analyzed by flow cytometry. $10 \mu$ l or $20 \mu$ of heatinactivated serum was incubated with washed S. pneumoniae WU2 cells in $100 \mu$ l of a reaction mixture achieving at a final concentration of $10^{8} \mathrm{cfu} / \mathrm{ml}$ at $37^{\circ} \mathrm{C}$ for $30 \mathrm{~min}$. The live bacteria were washed once with PBS and then incubated with $10 \%$ fresh normal mouse serum as the source of complement in gelatin veronal buffer with $\mathrm{Ca}^{2+}$ and $\mathrm{Mg}^{2+}$ (Sigma, St. Louis, MO) at $37^{\circ} \mathrm{C}$ for $30 \mathrm{~min}$. After washing, live bacteria were incubated with $100 \mu \mathrm{l}$ of fluorescein isothiocyanate-conjugated anti-mouse C3 IgG (MP Biomedicals, Solon, OH) in PBS for $30 \mathrm{~min}$ on ice. The samples were fixed with $2 \%$ formaldehyde after two washing steps and stored at $4^{\circ} \mathrm{C}$. Flow cytometry analysis was conducted using a FACSCalibur apparatus (Becton Dickinson), and 10,000 gated events were recorded.

\section{Fatal pneumococcal pneumonia model}

To determine the protective effects of nasal immunization with PspA plus each TLR agonist, S. pneumoniae WU2 strain at a dose of 2.0 $\times 10^{7} \mathrm{cfu}\left(3 \times \mathrm{LD}_{50}\right)$ suspended in $30 \mu \mathrm{l}$ of sterile saline was intranasally administered to both the immunized and untreated mice 2 weeks after the last immunization. The 2 -week interval between the last immunization and the bacterial challenge was maintained to avoid the influence of each TLR agonist on pulmonary defense, since some TLRs are involved in the innate immune response to $S$. pneumoniae [14-16]. The blood or lungs were aseptically removed from mice that had been anesthetized or euthanized with pentobarbital at $24 \mathrm{~h}$ post-bacterial challenge. The lung tissue was homogenized in $2 \mathrm{ml}$ of sterile saline per whole lung tissue prior to culturing. To prevent coagulation, the blood was collected in tubes containing heparin. Quantitative bacterial cultures of blood or lung tissues were performed on horse blood agar. Mortality was monitored for 16 days following the pneumococcal challenge.

\section{Statistics}

Statistical analyses were performed using one-way ANOVA and Tukey's multiple comparison method for antibody titers and bacterial clearance [17], and a log rank (Mantel-Cox) test for analysis of the survival curve with Graphpad Prism (GraphPad Software, Sandiego, 
Citation: Piao Z, Oma K, Ezoe H, Akeda Y, Tomono K, et al. (2011) Comparative Effects of Toll-Like Receptor Agonists on a Low Dose PspA Intranasal Vaccine against Fatal Pneumococcal Pneumonia in Mice. J Vaccines Vaccin 2:113. doi:10.4172/2157-7560.1000113

CA). Data were considered to be statistically significant if the $p$-values were less than 0.05 .

\section{Results}

Immune responses to PspA in mice after nasal immunization with a low dose of PspA plus TLR agonists

While no PspA-specific IgG was detected in sera of mice that had been immunized nasally with PspA alone, increased high levels of PspAspecific IgG were detected in sera of mice that had been immunized nasally with PspA plus and either LPS, Pam3CSK4, Poly(I:C) or CpG 1826 (Figure 1A). The levels of PspA-specific IgG in the sera of mice immunized PspA plus LPS were significantly higher than those of mice that had been immunized with PspA plus and either Poly(I:C) $(p<0.01)$ or CpG $1826(p<0.01)$. No significant difference was found among the levels of PspA-specific IgG among mice that had been immunized nasally PspA plus Pam3CSK4, Poly(I:C) or CpG 1826.
The levels of PspA-specific IgG in the BAL fluids and NWs of mice that had been nasally immunized with PspA plus the LPS were $5.17 \pm$ 0.98 and $1.67 \pm 1.03$, respectively. In contrast, negligible levels of PspAspecific IgG were induced in the BAL fluids and NWs from mice that had been nasally immunized PspA plus Pam3CSK4, Poly(I:C) and CpG 1826. PspA-specific IgA was not detected in the BAL fluid or NWs from these mice that had been immunized nasally with PspA plus the TLR agonist.

No PspA-specific IgG1 or IgG2a was detected in sera of mice that had been immunized nasally with PspA alone (Figure 1B). In contrast, increased levels of PspA-specific IgG1 or IgG2a were found in mice that had been immunized nasally with PspA plus the TLR agonist. The levels of PspA-specific IgG1 in sera of mice that had been immunized nasally with PspA plus LPS were significantly higher than those of mice that were immunized nasally with PspA plus Pam3CSK4 $(p<0.05)$, PspA plus Poly(I:C) $(p<0.01)$ or PspA plus CpG $1826(p<0.01)$. No
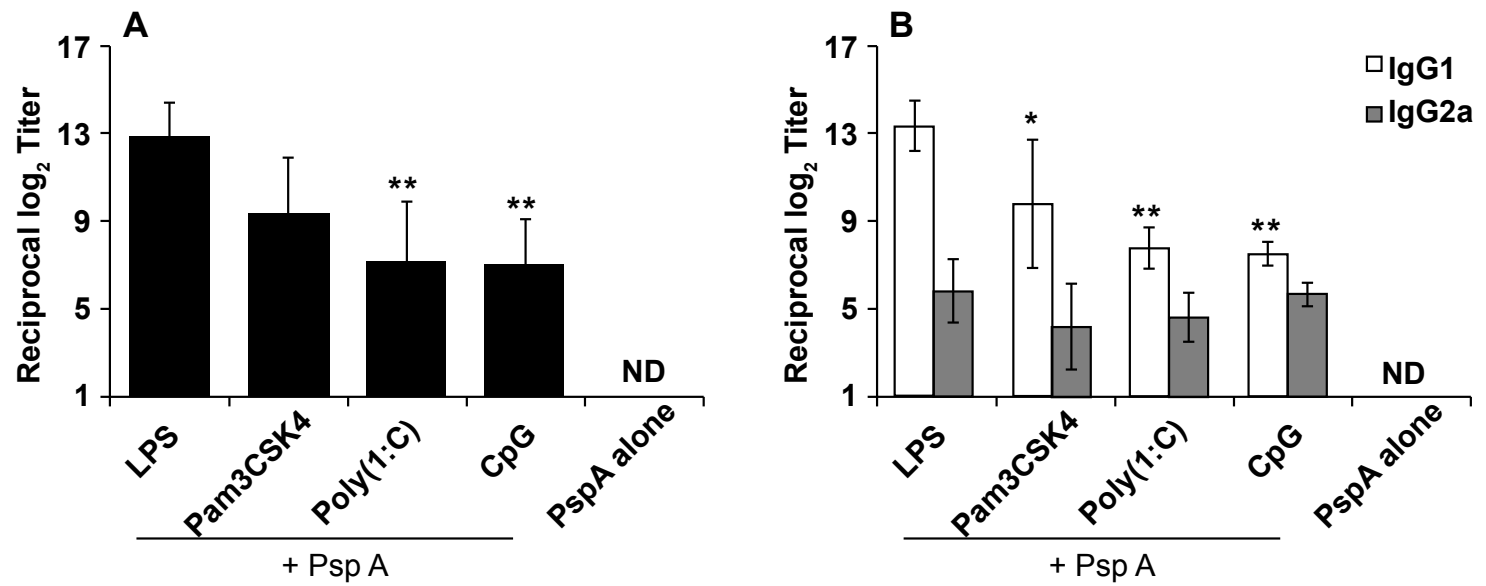

Figure 1: Induction of PspA-specific IgG (closed bars)(A), PspA-specific IgG1 (open bars) and IgG2a (gray bars) (B) in serum by intranasal immunization with either PspA plus each TLR agonist or PspA alone. Mice were nasally immunized three times at weekly intervals with $10 \mu \mathrm{g}$ of TLR agonist and $0.1 \mu \mathrm{g}$ of PspA. One week after the final immunization, the mice were euthanized to obtain serum, and PspA-specific antibody titers were determined using ELISA. The results are expressed as the mean \pm S.D. for six mice per group. ${ }^{*} p<0.05,{ }^{* *} p<0.01$, when compared with mice that were nasally immunized PspA plus LPS. ND, not detected.
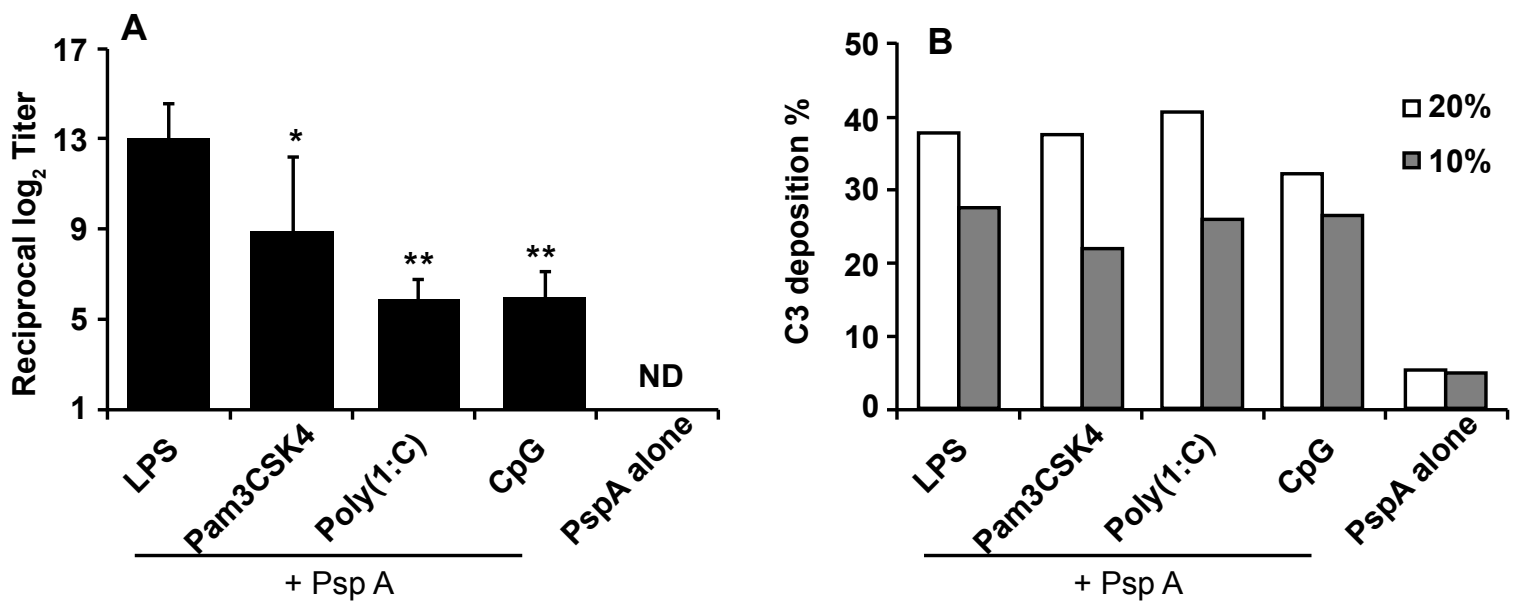

Figure 2: Binding of $\operatorname{lgG}$ antibodies (A) and C3 deposition (B) on the S. pneumoniae WU2 cell surface in the presence of sera from immunized and control mice. Binding The levels of IgG were analyzed by whole cell ELISA, and the C3 deposition were analyzed by flow cytometry using $S$. pneumoniae WU2 cells which were incubated with $20 \%$ (open bars) or $10 \%$ (gray bars) of pooled sera from mice that were immunized by intranasal immunization with either PspA plus each TLR agonist or PspA alone. The percentage of fluorescent bacteria (greater than 10 fluorescence intensity units) is shown as C3 deposition for each sample. ${ }^{*} p<0.05$, ${ }^{* *} p<0.01$, when compared with mice that were nasally immunized PspA plus LPS. The results are expressed as the mean \pm S.D. for six mice per group. ND, not detected. 

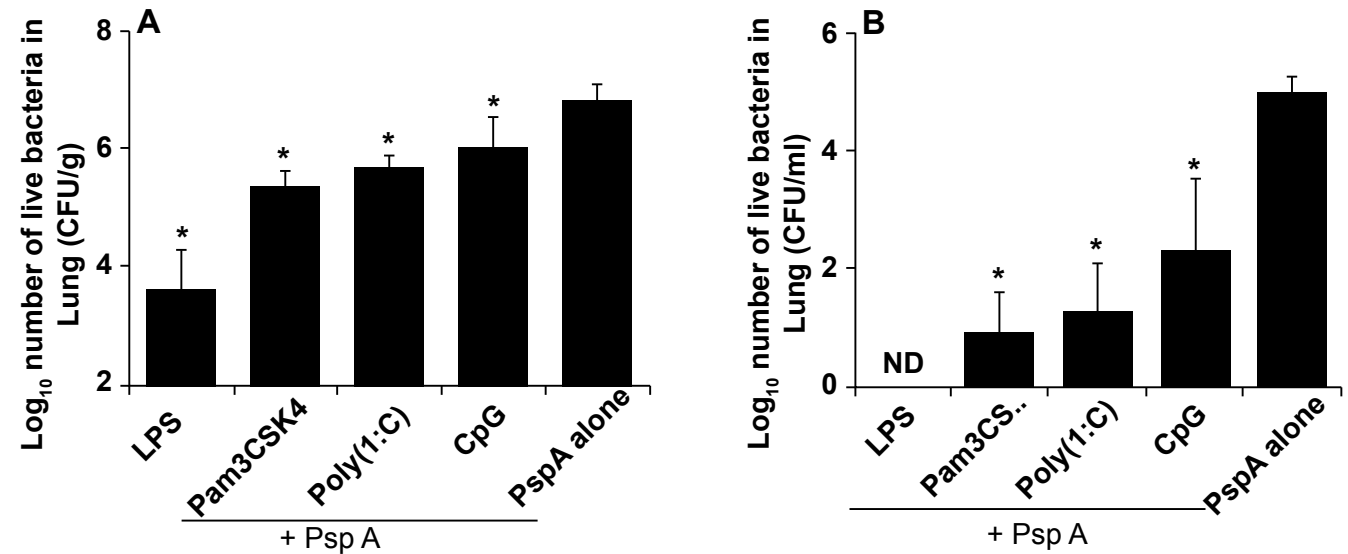

Figure 3: The effect of intranasal immunization with PspA plus each TLR against on the bacterial densities in Lung tissue (A) and blood (B) at $24 \mathrm{~h}$ post-challenge with the S. pneumoniae WU2 strain. An invasive dose of $2 \times 10^{7} \mathrm{cfu} /$ mouse was nasally administered to mice that had been previously immunized with either PspA plus each TLR agaonist or PspA alone. Mice were euthanized to obtain lung tissues or blood from infected mice at the indicated time-point after bacterial challenge, and quantitative bacterial cultures of lung tissue or blood were performed. Values represent the $\log _{10} \mathrm{cfu} / \mathrm{g}$ or $\log _{10} \mathrm{cfu} / \mathrm{ml}$ (mean $\pm \mathrm{S}$.D.) for six mice per group. ${ }^{*} p<0.0001$, when compared with mice that were nasally immunized with PspA alone.

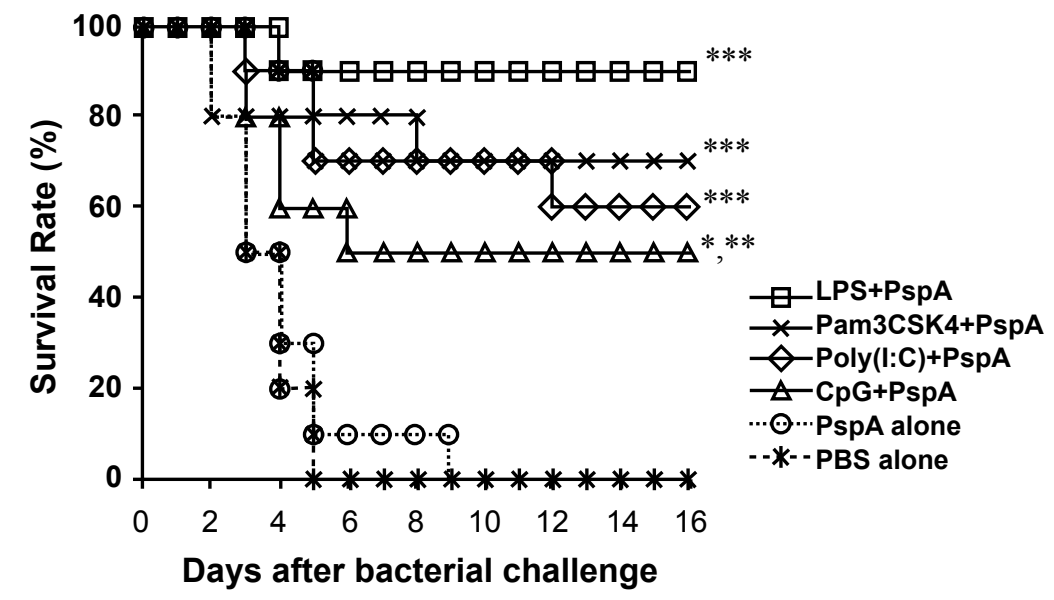

Figure 4: Survival of mice that were immunized nasally with a low dose of PspA plus each TLR agonist, a low dose of PspA alone and PBS alone after pneumococcal pneumonia. Immunized mice were intranasally challenged with $2 \times 10^{7} \mathrm{cfu}$ of pneumococcal strain WU2, and the survival of the mice was monitored for 16 days. Results were examined by a Kaplan-Meier survival curve analysis for ten mice per group. ${ }^{*} p<0.05$, when compared with mice that were nasally immunized PspA alone. ${ }^{* *} p<$ 0.01 , when compared with mice that were nasally immunized PBS alone. ${ }^{* *} p<0.001$, when compared with mice that were nasally immunized PspA alone or PBS alone.

significant difference was found in PspA-specific IgG1 levels among the sera of mice that were nasally immunized with PspA plus Pam3CSK4, Poly(I:C) or CpG 1826. No significant difference was found in PspAspecific IgG2a among sera of mice that were immunized nasally with PspA plus the TLR agonist. The mean ratios of PspA-specific IgG1 titers to PspA-specific IgG2a titers were 2.26 for LPS, 2.33 for Pam3CSK4, 1.68 for Poly(I:C), and 1.32 for CpG 1826, respectively.

\section{IgG binding and $\mathrm{C} 3$ deposition on the bacterial surface}

While no binding of mouse $\operatorname{IgG}$ was observed in the case of bacteria treated with sera from mice that were immunized nasally with PspA alone, the levels of mouse IgG found on bacteria that were treated with sera from mice immunized nasally with the PspA plus LPS, Pam3CSK4, Poly(I:C) or CpG 1826 (Figure 2A) was increased. The levels of mouse IgG were significantly higher in sera from mice that were immunized nasally PspA plus LPS compared to mice that were nasally immunized with PspA plus Pam3CSK4 ( $p<0.05$ ), PspA plus poly (I:C) or PspA plus CpG $1826(p<0.01)$. These levels of mouse IgG in serum from mice that were immunized nasally with PspA plus the TLR agonist closely corresponded with the levels of PspA-specific IgG induced in serum. The frequencies of C3 depositions were substantially increased in bacteria that had been pretreated with $10 \%$ and $20 \%$ sera from mice that were immunized nasally with PspA plus each of the TLR agonists, compared with those of mice that were immunized nasally with PspA alone (Figure 2B). No differences were found in the frequencies of $\mathrm{C} 3$ deposition on bacteria that were pretreated with sera from mice nasally immunized with PspA plus each TLR agonist.

\section{Bacterial clearance from the lungs and the blood}

The bacterial densities (mean \pm S.D. for $\log _{10}$ cfu / g) reached $6.83 \pm 0.26$ in the lung and $4.89 \pm 0.3$ in blood from mice that were immunized nasally with PspA alone at $24 \mathrm{~h}$ post-challenge (Figure $3 \mathrm{~A}$ and $3 \mathrm{~B}$ ). Significant decreases were found in bacterial density in the lungs of mice that were immunized nasally with PspA plus either 
LPS, Pam3CSK4, Poly(I:C) or CpG 1826 compared with mice that were immunized nasally with the PspA alone $(p<0.0001)$. No significant differences were found in the bacterial densities in the lung among mice immunized nasally with PspA plus LPS, Pam3CSK4, Poly(I:C) or CpG 1826 (Figure 3A). Significant decreases were also found in blood from mice that were immunized nasally with PspA plus either Pam3CSK4, Poly(I:C) or CpG 1826 compared with mice that were immunized nasally with the PspA alone $(\mathrm{p}<0.0001)$. No bacteria were detected in blood samples from any of the mice that were immunized nasally with PspA plus LPS agonist. No significant differences were found among mice immunized nasally with PspA plus LPS, Pam3CSK4, Poly(I:C) or CpG 1826 (Figure 3B).

\section{Protection by PspA plus each TLR vaccine against fatal pneumococcal pneumonia}

As shown in Figure 4, the Kaplan-Meier analysis demonstrated significant protection as evidenced by the mean survival rate for mice that were immunized nasally with PspA plus each TLR agonist compared with mice that were immunized nasally with PspA alone or PBS alone. The survival rate was $90 \%$ for mice immunized nasally with the PspA plus LPS agonist ( $p<0.0001$ for PspA alone or PBS alone), $70 \%$ for mice immunized nasally with PspA plus Pam3CSK4 agonist ( $p=0.0008$ for PspA alone or $p=0.0006$ for PBS alone), $60 \%$ for mice immunized nasally with PspA plus Poly(I:C) agonist $(p=0.0005$ for PspA alone or $p=0.0003$ for PBS alone), $50 \%$ for mice immunized nasally with PspA plus CpG 1826 agonist ( $p=0.0127$ for PspA alone or $p=0.0062$ for PBS alone), respectively. No significant differences were found among mice that were immunized nasally with PspA plus LPS, Pam3CSK4, Poly(I:C) or CpG 1826.

\section{Discussion}

The findings reported herein provide a demonstration of the protective effects of the nasal vaccination of a low dose of PspA plus each TLR agonist against a fatal model of pneumococcal pneumonia with serotype 3 S. pneumoniae WU2. Nasal vaccination of a low dose of PspA plus each TLR agonist induced a high level of PspA-specific IgG in the serum and a low level of PspA-specific IgG in the airways of mice. The binding of PspA-specific IgG in sera resulted in an increase in C3 deposition on the bacterial surfaces. Subsequently, the bacterial densities in the lung tissues and blood were significantly decreased in mice that were immunized nasally with PspA plus TLR agonist, compared with the values for mice immunized nasally with a low dose of PspA alone. The reduction in bacterial densities in lung tissues could be explained by the sufficient extravasation of PspA-specific IgG into the alveolar space of mice that were immunized nasally with a low dose of PspA plus each TLR agonist [18]. Bacterial invasion into the blood circulation was readily suppressed by PspA-specific IgG in sera of mice immunized nasally with a low does of PspA plus each of the TLR agonists. The survival of infected mice that were immunized nasally with a low dose of PspA plus TLR agonist was significantly increased compared with those of mice that were immunized nasally with a low dose of PspA alone. These findings are in contrast with findings reported in our previous study, showing no significant difference in the survival of infected mice between nasal immunization with a high dose $(2.5 \mu \mathrm{g})$ of PspA plus TLR agonist and a high dose of PspA alone in a fatal pneumonia model using the WU2 strain [12]. The findings reported herein on the effect of a low dose of PspA plus TLR agonist also suggest possibilities for the development of a cost-effective PspA intranasal vaccine with the goal of preventing a fatal pneumonia.

We recently reported on the pivotal role of PspA-specific IgA on the bacterial clearance of a less virulent serotype $19 \mathrm{~F}$ strain in the upper airway in a mouse model of bacterial colonization [19]. Since PspA-specific IgG was shown to be sufficient for protecting mice against a fatal bacteremic pneumonia caused by a virulent serotype 3 strain in this study, PspA-specific IgA may not be essential for invasive pneumococcal infections, such as bacteremic pneumonia.

While the rank orders of PspA-specific IgG induced in sera were LPS > Pam3CSK4 > Poly(I:C) and CpG 1826, no difference was found in the frequency of $\mathrm{C} 3$ deposition on bacterial surfaces in immune sera induced by a low dose of PspA plus each TLR agonist in this study. This discrepancy between the levels of PspA-specific IgG and the frequencies of $\mathrm{C} 3$ deposition may be explained by the similar ratios of PspA-specific IgG1 titers to IgG2a titers (1.32 to 2.33) among mice that were immunized nasally with a low dose of PspA plus each TLR agonist in this study, because the $\mathrm{C} 3$ binding activity of the IgG2a isotype is superior to those of other IgG isotypes [20]. Although antibacterial effects in the lung and blood were the highest in mice that were immunized nasally with a low dose of PspA plus LPS than those in mice immunized nasally with a low dose of PspA plus the other TLR agonists at $24 \mathrm{~h}$ post-infection, no significant difference was found in the survival rate of mice immunized nasally with a low dose of PspA plus each TLR agonist. This discrepancy may be explained by subsequent bacterial growth in the lungs and blood in mice that had been immunized nasally with a low dose of PspA plus LPS as well as mice immunized nasally with a low dose of PspA plus the other TLR agonists later than $24 \mathrm{~h}$ post-infection.

Despite similar C3 binding activities of immune sera, the rank orders for the survival rates of the immunized mice were LPS $>$ Pam3CSK $4>$ Poly(I:C) and CpG 1826, and were in agreement with those of plasma levels of PspA-specific IgG reported in this study. Although LPS or Pam3CSK4 demonstrated a superior adjuvant effect among the TLR agonists tested in this study, these bacterial products are highly toxic to humans. In contrast, a synthetic analogue of a dsRNA compound, such as Poly (I:C) or Poly I:PolyC12 U (Ampligen ${ }^{\mathrm{R}}$ ), or CpG ODN would be expected to be applicable as a safe mucosal adjuvant in humans [21,22].

In conclusion, the data presented here provide evidence to indicate that intranasal immunization with a vaccine containing a low dose of PspA plus each TLR agonist elicited a high level of systemic PspAspecific IgG, and was capable of preventing the development of fatal pneumococcal pneumonia in mice. An intranasal administration of each TLR agonist in combination with a low dose PspA significantly increased the survival rates of the infected mice in the following order: LPS > Pam3CSK4 > Poly(I:C) and CpG ODN 1826. This study confers an important insight regarding strategies for a cost-effective PspA protein-based vaccine against invasive pneumococcal infections.

\section{Acknowledgments}

We are grateful to Dr. DE Briles and Dr. SK Hollingshead from the University of Alabama at Birmingham, for providing the pneumococcal strain and the recombinant plasmid, pUAB055, used in this study. This work was supported by the Program for Promotion of Fundamental Studies in Health Sciences of the National Institute of Biomedical Innovation (NIBIO), and by the Biomedical Cluster Kansai project, which is promoted by the Regional Innovation Cluster Program and subsidized by the Japanese Government, by the Global Center of Excellence Programs of Osaka University, and by Grants-in-Aid from the Ministry of Health, Labor and Welfare of Japan for "Mechanisms, epidemiology, prevention and control of acute respiratory infection".

\section{References}

1. Garau J (2002) Treatment of drug-resistant pneumococcal pneumonia. Lancet Infect Dis 2: 404-415. 
Citation: Piao Z, Oma K, Ezoe H, Akeda Y, Tomono K, et al. (2011) Comparative Effects of Toll-Like Receptor Agonists on a Low Dose PspA Intranasal Vaccine against Fatal Pneumococcal Pneumonia in Mice. J Vaccines Vaccin 2:113. doi:10.4172/2157-7560.1000113

2. Butler JC, Shapiro ED, Carlone GM (1999) Pneumococcal vaccines: history, current status, and future directions. Am J Med 107: 69S-76S.

3. Singleton RJ, Hennessy TW, Bulkow LR, Hammitt LL, Zulz T, et al. (2007) Invasive pneumococcal disease caused by nonvaccine serotypes among Alaska native children with high levels of 7 -valent pneumococcal conjugate vaccine coverage. JAMA 297: 1784-1792.

4. Hicks LA, Harrison LH, Flannery B, Hadler JL, Schaffner W, et al. (2007) Incidence of pneumococcal disease due to non-pneumococcal conjugate vaccine (PCV7) serotypes in the united states during the era of widespread PCV7 vaccination, 1998-2004. J infect Dis 196: 1346-1354.

5. Briles DE, Tart RC, Swiatlo E, Dillard JP, Smith P, et al. (1998) Pneumococcal diversity: considerations for new vaccine strategies with emphasis on pneumococcal surface protein A (PspA). Clin Microbiol Rev 11: 645-657.

6. Tai SS (2006) Streptococcus pneumoniae protein vaccine candidates:properties, activities and animal studies. Crit Rev Microbiol 32: 139-153.

7. Briles DE, Hollingshead SK, King J, Swift A, Braun PA, et al. (2000) Immunization of humans with recombinant pneumococcal surface protein $\mathrm{A}(\mathrm{rPspA})$ elicits $\mathrm{Abs}$ that passively protect mice from fatal infection with Streptococcus pneumoniae Bearing Heterologous PspA. J Infect Dis 182: 1694-1701.

8. Nabors GS, Braun PA, Herrmann DJ, Heise ML, Pyle DJ, et al (2000) Immunization of healthy adults with a single recombinant pneumococcal surface protein A (PspA) variant stimulates broadly cross-reactive Abs to heterologous PspA molecules. Vaccine 18: 1743-1754.

9. Mcdaniel LS, Mcdaniel DO, Hollingshead SK, Briles DE (1998) Comparison of the PspA sequence from Streptococcus pneumoniae EF5668 to the previously identified PspA sequence from strain Rx1 and ability of PspA from EF5668 to elicit protection against pneumococci of different capsular types. Infect Immun 66: 4748-4754.

10. Mcdaniel LS, Ralph BA, Mcdaniel DO, Briles DE (1994) Localization of protection-eliciting epitopes on PspA of Sterptococcus pneumoniae between amino acid residues 192 and 260. Microb Pathogen 17: 323-337.

11. Freytag LC, Clements JD (2005) Mucosal adjuvants. Vaccine Rev 23: 1804-1813.

12. Oma K, Zhao J, Ezoe H, Akeda Y, Koyama H, et al. (2009) Intranasal immunization with a mixture of PspA and a Toll-like receptor agonist induces specific Abs and enhances bacterial clearance in the airways of mice. Vaccine 27: 3181-3188.

13. Kurita S, Koyama J, Onizuka S, Motomura K, Watanabe $\mathrm{H}$, et al. (2006) Dynamics of dendritic cell migration and the subsequent induction of protective immunity in the lung after repeated airway challenges by nontypeable Haemophilus influenzae outer membrane protein. Vaccine 24: 5896-5903.

14. Malley R, Henneke P, Morse SC, Cieslewicz MJ, Lipstich M, et al. (2003) Recognition of pneumolysin by Toll-like receptors 4 confers resistance to pneumococcal infection. Proc Natl Acad Sci 100: 1966-1971.

15. Albiger B, Dahlberg S, Sandgren A, Wartha F, Beiter K, et al. (2007) Tolllike receptor 9 acts at an early stage in host defense against pneumococcal infection. Cell Microbiol 9: 633-644.

16. McNeela EA, Burke A, Neill DR, Baxter C, Fernandes VE, et al. (2010) Pneumolysin activates the NLRP3 inflammasome and promotes proinflammatory cytokines independently of TLR4. PLoS Pathogens 6: e1001191.

17. Stoline MR (1981) The status of multiple comparisons: simultaneous estimation of all pairwise comparisons in one-way ANOVA designs. Am Stat 35: 134-135.

18. Sato S, Ouellet N, Pelletiter I, Shimard M, Rancourt A, et al. (2002) Role of galectin-3 as an adhesion molecule for neutrophil extravasation during Streptococcus pneumonia. J Immunol 168: 1813-1822.

19. Fukuyama Y, King JD, Kataoka K, Kobayashi R, Gilbert RS, et al. (2010) Secretory-IgA antibodies play an important role in the immunity to Streptococcus pneumoniae. J Immunol 185: 1755-62.

20. Oishi K, Koles NL, Guelde G, Pollack M (1992) Antibacterial and protective properties of monoclonal antibodies reactive with Escherichia coli 0111:B4 lipopolysaccharide: relation to antibody isotype and complement-fixing activity. $\mathrm{J}$ Infect Dis 165: 34-45.

21. Thompson KA, Strayer DR, Salvato PD, Thompson CE, Klimas N, et al. (1996) Results of a double-blind placebo-controlled study of the double-stranded RNA drug Polyl:Poly $\mathrm{C}_{12} \mathrm{U}$ in the treatment of HIV infection. Eur $\mathrm{J}$ Clin Microbiol Infect Dis 15: $580-587$.

22. Kodama S, Abe N, Hirano T, Suzuki M (2006) Safety and efficacy of nasa application of $\mathrm{CpG}$ oligodeoxynucleotide as a mucosal adjuvant. Laryngoscope 116: 331-335. 